\title{
NARROWBAND BEAM LOADING COMPENSATION IN THE FERMILAB MAIN INJECTOR ACCELERATING CAVITIES*
}

\author{
J. Dey ${ }^{\dagger}$ J. Steimel, J. Reid, FNAL*, Batavia, IL 60510, USA
}

\begin{abstract}
A narrowband beam loading compensation system was installed for the Main Injector Accelerating Cavities. This feedback operates solely on the fundamental resonant mode of the cavity. This paper describes modifications to the high level Radio Frequency system required to make the system operational. These modifications decreased the effect of steady-state beam loading by a factor of 10 and improved the reliablity of paraphasing for coalescing.
\end{abstract}

\section{INTRODUCTION}

During the course of Run II at Fermilab, the main injector will perform many RF voltage amplitude manipulations for various beam gymnastics. These include coalescing, slip-stacking, bunch rotation, and deceleration. Most of the manipulations require very precise amplitude control of the main accelerating cavities. In some cases, the amplitude required is significantly less than the voltage generated by the beam current itself. In other cases, the total voltage seen by the beam through the accelerating cavities should be eliminated.

The amount of beam-generated voltage allowed for reliable slip-stacking and deceleration is quite low, and the present high level RF system will not meet the specifications.

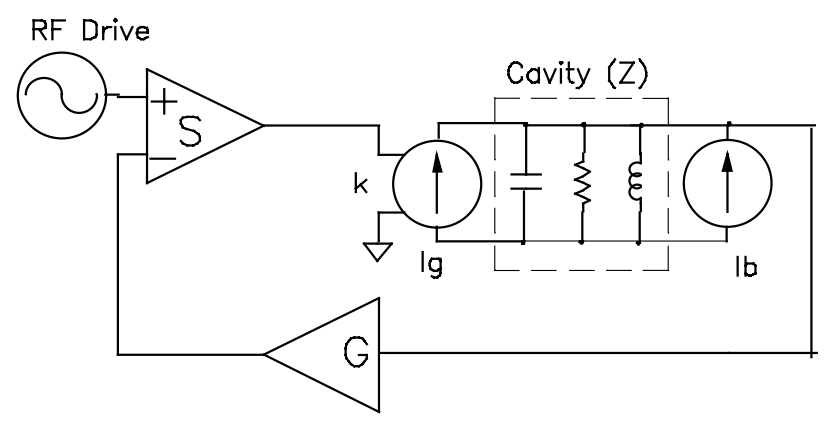

Figure 1: Direct RF Feedback Block Diagram. G is the feedback gain, and $\mathrm{k}$ is the tube transconductance.

One way to overcome this limitation is with direct RF feedback around the cavities. The design of direct RF feedback that meets the specifications of slip-stacking is complicated and requires changes in the way that the high level RF system operates. In order to discover the

\footnotetext{
* Operated by Universities Research Association, Inc. for the U.S Department of Energy under contract DE-AC02-76CH03000.

†dey@fnal.gov
}

changes necessary to accommodate RF feedback, a simple feedback system was commissioned. This simple system not only revealed necessary changes to the high level system, it also provided improved performance for other aspects of main injector operation.

\section{DIRECT RF FEEDBACK}

Implementation of direct RF feedback is relatively straightforward. Figure 1 illustrates the basic concept. A signal that is linearly related to the voltage at the cavity gap is combined with the low level drive of the power amplifier[1]. The loop will create a negative feedback system with the proper sign and delay.

\subsection{Theory}

The feedback loop will reduce the effective impedance seen by the beam (Eq. 1) and alter the voltage transfer function as shown in Equation 2. Because this is a closed loop system, the open loop response must not violate Nyquist stability criteria. The open loop response includes the cavity impedance and any electrical delay associated with the round trip distance from the feedback summing point to the cavity. The loop gain limit (Eq. 3) for the main injector $53 \mathrm{MHz}$ RF system is about $26 \mathrm{~dB}$ for a round trip delay of about $300 \mathrm{~ns}$.

$$
\begin{aligned}
& \frac{V r f}{I b}=\frac{Z(\omega)}{1+k G(\omega) Z(\omega)} \\
& \frac{V r f}{V i n}=\frac{k Z(\omega)}{1+k G(\omega) Z(\omega)} \\
& |G(\omega)|<\frac{1}{\frac{4}{Q} \tau_{D} f_{r f}}
\end{aligned}
$$

\subsection{Filter Design}

The filter consists of a superheterodyne structure with low pass components (Figure 2). The roll-off frequency of these low pass components is much higher than the cavity half bandwidth. Therefore, the cavity dominates the filter response of the loop. The superheterodyne structure maintains the $180^{\circ}$ phase shift through the loop at the resonant frequency. The round trip delay would normally cause the phase to change as the accelerator frequency changed, but with the mixer local oscillator inputs properly delayed, the phase remains fixed. 


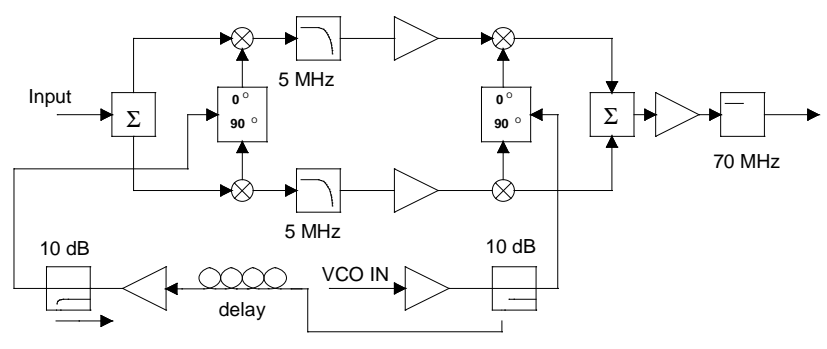

Figure 2: Beam Loading Compensation Filter Module

\section{HIGH LEVEL RF SYSTEM}

The high level RF system consists of 18 identical 53 Mhz accelerating stations spaced $\mathrm{N}^{*} \lambda / 2$ apart installed at MI-60 and operating at harmonic number 588. Each station consists of a Ferrite tuned accelerating cavity, 200 Kwatt cathode driven power amplifier mounted directly to accelerating cavity, 4 Kwatt wideband solid state MOSFET driver amplifier, 30 Kvolt series tube modulator, Ferrite Bias Supply, and a common anode power supply for six stations. Also included are global and local timing channels, analog curve generators, and a VME based data acquisition system. Feedback control circuits are contained in NIM style modules.

\subsection{Cavity}

\begin{tabular}{|l|l||}
\hline Peak Accelerating Voltage: & 240 Kvolts / Cavity \\
\hline Q @ 53.1 Mhz: & $\sim 5000$ \\
\hline R Shunt: & $\sim 520 \mathrm{~K}$ \\
\hline R/Q & 104 \\
\hline Tuning Range & $52.8 \mathrm{Mhz}$ to $53.1 \mathrm{Mhz}$ \\
\hline Step-up Ratio & $\sim 12: 1$ \\
\hline
\end{tabular}

Table 1: Nominal Main Injector RF Cavity parameters

The accelerating cavity may be thought of as two quarter wave TEM mode coaxial reentrant resonators back to back. The cavity is tuned through its range by inductively coupling energy from the high current region into externally mounted ferrite tuners. The cavities required no modifications for implementing direct rf feedback.

\subsection{Power Amplifier}

The 200 Kwatt rf power amplifiers were new for the Main Injector and utilize a Y-567 tetrode that is cathode driven. They are rf grounded-grid with the operating point efficiently regulated with a dc grid bias voltage and variable anode voltage. At the present time we gate the grid bias from -400 volts to -275 volts during the $\mathrm{rf}$ on time while programming the DC anode voltage to give the desired accelerating voltage.

RF is supplied to the base of the cathode circuit by four parallel $50 \mathrm{ohm}$ Heliax cables from the output combiner of the solid state amplifier. The cathode resonator has a $\mathrm{Q}$ of 10. The low $\mathrm{Q}$ is accomplished by tapping the cathode resonant structure with two 50 terminations tapped for a 4:1 impedance transformation at the cathode (100 Ohms).

\subsection{Solid State Driver}

\begin{tabular}{|l|l||}
\hline Frequency: & $30 \mathrm{Mhz}$ to $80 \mathrm{Mhz}$ \\
\hline Gain: & $50 \mathrm{db}$ \\
\hline Gain Flatness: & $+/-1 \mathrm{db}$ \\
\hline Phase Delay & $24 \mathrm{nSec}$ \\
\hline RF output Power & 4 Kwatts \\
\hline Cooling & 10 gpm of LCW \\
\hline
\end{tabular}

Table 2: Abbreviated Solid State Amplifier Specification

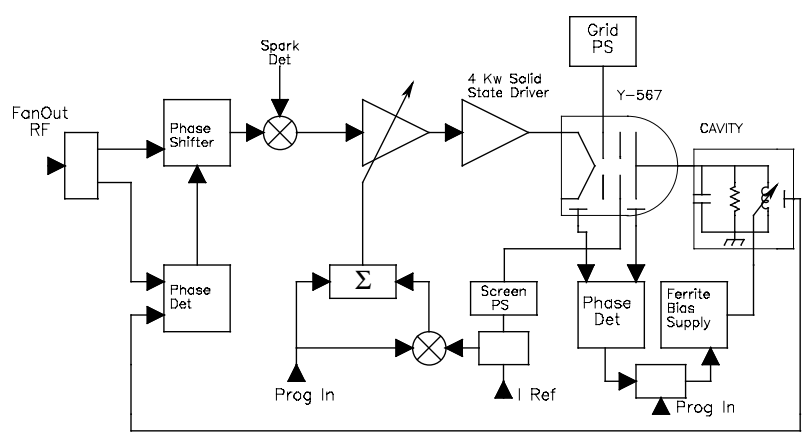

Figure 3: Block Diagram of High Level System before direct rf feedback was employed

The solid state driver amplifier consists of four 1 Kwatt rf modules, a four way combiner, control unit, and 48 Volt power supply. A variable gain $\mathrm{rf}$ amplifier is employed for programming rf level to the 1 Kwatt modules in response to an input program of 0 to 10 volts. Overall amplitude regulation is provided by requiring constant Y567 screen current which is used as feedback to the solid state driver's variable gain amplifier (Figure 3).

\subsection{Series Tube Modulator}

The series tube modulator was designed new for the Main Injector and requires no modifications. It has a bandwidth of $100 \mathrm{Khz}$ and produces a 0 to $30 \mathrm{Kvolt}$ output in response to an input program of 0 to 10 volts. It has a fast acting blocking circuit to provide isolation from the common anode supply should the power amplifier tube arc. 


\subsection{Ferrite Bias Supply}

The Ferrite Bias Supply is a 12 phase SCR controlled raw supply with an active filter. Typical operating currents are $\sim 50$ amps at injection and 500 amps at flattop. However these will shift upwards as the duty factor increases to compensate for heating in the cavity. Large signal slew rate is 250,000 amps per second with small signal response around $1 \mathrm{Khz}$.

\section{HIGH LEVEL MODIFICATIONS}

Certain modifications of the high level system were necessary for robust operation of the beam loading compensation system. Most of the modifications were performed on the front end of the solid state driver (Figure 4). Other modifications involved disabling feedback loops for zero RF voltage operation.

\subsection{Solid State Driver}

For stability reasons, it is beneficial to have the amplitude control for the driver upstream of the summing junction of the feedback loop. This keeps the loop gain constant. According to the new transfer function (Eq. 2), the gain in front of the summing junction must be increased by the total feedback loop gain in order to maintain the same voltage level in the cavities. Therefore, a fixed amplifier was added downstream of the variable gain amplifier with enough dynamic range to handle the new drive conditions.

Because the loop has a finite delay, there is a voltage overshoot to a step change in the RF drive. Examples of

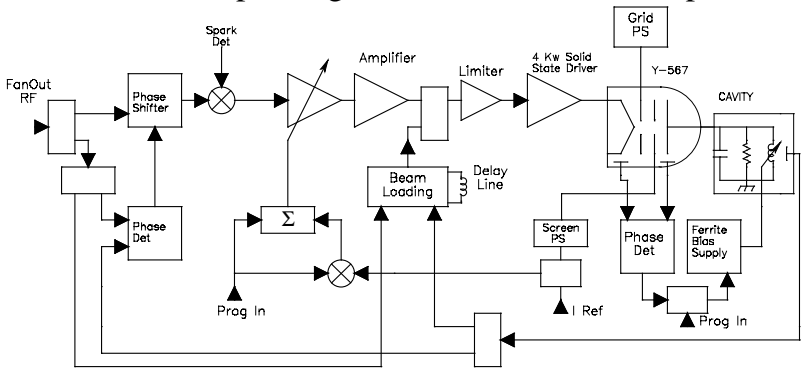

Figure 4: Block Diagram of High Level System with Beam Loading Compensation

step changes include gating on both the drive and transition jump. The overshoot seen by the solid state driver is proportional to the drive level times the open loop gain at the drive frequency. An open loop gain of 10 causes an overshoot that would require the solid state driver to handle a transient input with 100 times the nominal power. The driver cannot handle this input without tripping on over power. A limiter is added to the output of the driver module to prevent overdriving of the solid state amplifier. This does not affect the loop gain during steady state operating conditions. It only slows the system response to fast changes in drive input.

\section{RESULTS}

Figure 5 shows the effect of the beam loading compensation on a single cavities response. The cavity has been effectively de-Qed by the feedback. . The amount of de-Qing and impedance reduction is proportional to the open loop gain of the system (Eq. 1). The open loop gain is 8.5 (Figure 6). Higher operational gain (to 26) was observed but left at a conservative 8.5 so we could study long term station reliability.

The feedback system also reduces the amount of signal variation due to differences in power tubes, tuning bias, and other high power variations.

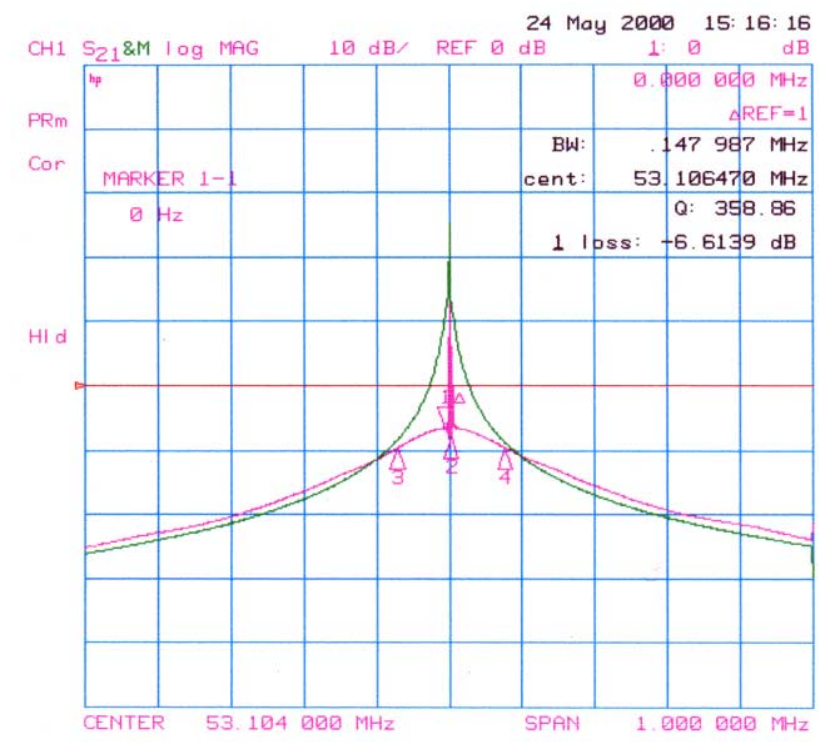

Figure 5: Main Injector frequency response with feedback (magenta) and without feedback (green).
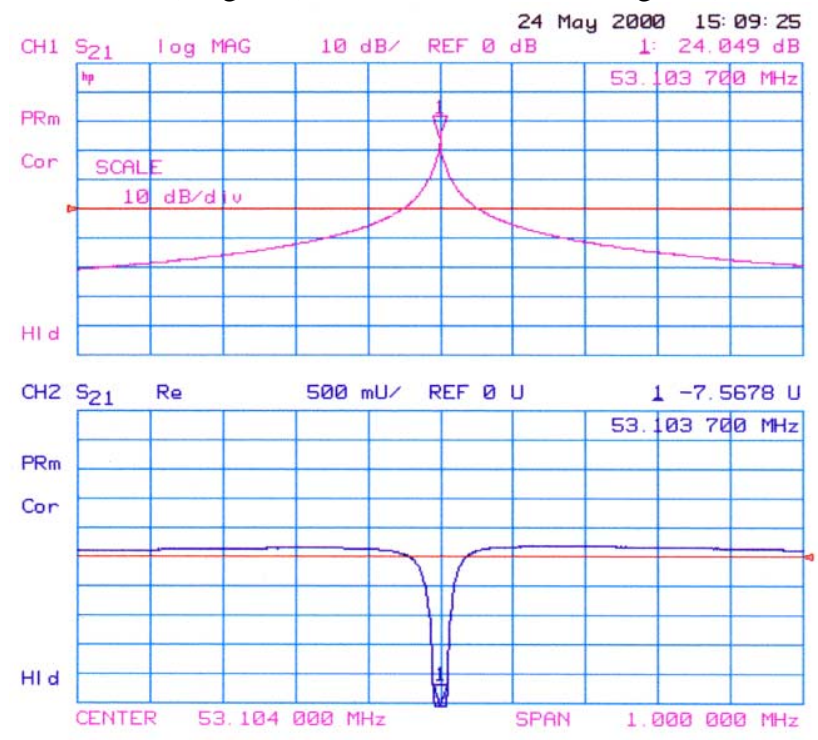

Figure 6: Main Injector Cavity open loop response

\section{REFERENCES}

[1] F. Pedersen, "RF Cavity Feedback," CERN, CH-121211 Geneva 23, Switzerland, Dec 1992. 